\title{
Radioguided thyroidectomy for follicular tumors: Multicentric experience
}

\author{
D. Parmeggiani a , C. Gambardella ${ }^{\text {c }}$, R. Patrone ${ }^{\text {c }}$, A. Polistena ${ }^{\text {d }}$, M. De Falco ${ }^{\text {b }}$, R. Ruggiero ${ }^{\text {a }}$, \\ R. Cirocchi ${ }^{d}$, A. Sanguinetti ${ }^{\mathrm{e}}$, V. Cuccurullo ${ }^{\mathrm{f}}$, M. Accardo ${ }^{\mathrm{g}}$, N. Avenia ${ }^{\mathrm{d}}$, G. Docimo ${ }^{\mathrm{a}}$, \\ S. Tolone ${ }^{\mathrm{a}}$, V. Bassi ${ }^{\mathrm{h}}$, L. Docimo a , G. Conzo ${ }^{\mathrm{c}, *}$ \\ a Unit of Surgery, Department of Medical, Surgical, Neurological, Metabolic and Aging Sciences, Second University of Naples (SUN), Naples, Italy \\ ${ }^{\mathrm{b}}$ V Unit of Surgery, Department of Surgical, Anesthesiological and Emergency Sciences, Second University of Naples (SUN), Naples, Italy \\ c VII Unit of Surgery, Department of Surgical, Anesthesiological and Emergency Sciences, Second University of Naples (SUN), Naples, Italy \\ ${ }^{\mathrm{d}}$ Endocrine Surgical Unit, University of Perugia, Italy \\ e U.O.C. of General Surgery, University Hospital of Terni, Italy \\ ${ }^{\mathrm{f}}$ Nuclear Medicine, Second University of Naples, Italy \\ ${ }^{g}$ Department of Morphopathology, Second University of Naples, Italy \\ h U.O.C. of Internal Medicine, S. Giovanni Bosco Hospital, ASL Na1 Centro, Naples, Italy
}

\section{H I G H L I G H T S}

- The diagnosis of thyroid nodular diseases requires an integrated approach.

- In case of "Follicular Lesions" findings the non-invasive technique is unable to distinguish carcinoma from follicular adenoma, then indicating the necessity of surgery to obtain a correct diagnosis.

\section{A R T I C L E I N F O}

\section{Article history:}

Received 31 December 2016

Received in revised form

29 March 2017

Accepted 29 March 2017

\section{Keywords:}

Thyroid cancer

Thyroid follicular neoplasm

99m Tc-MIBI scintigraphy

MIBI uptake

Radioguided surgery

Thyroidectomy

\begin{abstract}
A B S T R A C T
Background: The diagnosis of thyroid nodular diseases requires an integrated approach that has been widely established over the past years. This strategy includes: ultrasonography (US) with; implemented Color-Power-Doppler, conventional scintigraphy also with positive indicators, specific pathological studies targeted by immunohistochemically-assays, and the fine needle; aspiration biopsy (FNAB), which, usually, in case of "Follicular Lesions" (10-20\%) findings is; unable to distinguish carcinoma from follicular adenoma, then indicating the necessity of surgery to; obtain a correct diagnosis. The aim of this study was to evaluate the role of the scintigraphy with; positive indicators, both preoperatively in diagnostic approach of the thyroid nodules and; intraoperatively as a guide to the extension of the surgical excision.

Methods: On 4482 Thyroidectomy performed, we selected 360 cases of follicular neoplasms (192; females and 168 males). In the preoperative phase, these patients underwent $99 \mathrm{~m}$ Tc-sestaMIBI; scintigraphy with both early $(10 \mathrm{~min})$ and late $(2 \mathrm{~h})$ image acquisition, which were later; compared to the ones obtained by image subtraction of means $99 \mathrm{~m}$ Tc-pertechnetate. Following the; sestamibi administration before intervention, we selected the most up-taking nodularity with the; assistance of a specific surgical probe (Neoprobe), quantifying uptake with relation to the surgical pathology, for an amount of 324 total thyroidectomies and 36 hemi thyroidectomies.

Results: In all cases of multinodular goiter the benign nodules showed an intraoperative low sestamibi uptake whereas follicular carcinomas showed both a high preoperative uptake and, as a; percentage, the highest values of intraoperative uptake; on the other hand, follicular adenomas had; both pre-and intraoperative mean values of uptake. On the contrary, papillary carcinomas only; showed a mild uptake.
\end{abstract}

Abbreviations: US, ultrasonography; FNAB, fine needle aspiration biopsy; LBC, liquid-based-cytology.

* Corresponding author. Department of Anesthesiologic, Surgical and Emergency Sciences, Second University of Naples- Italy, Via Sergio Pansini 5, 80131, Naples, Italy.

E-mail address: giovanni.conzo@hotmail.it (G. Conzo). 
Conclusions: Preoperative sestamibi scintigraphy confirmed its importance in improving the information obtained through different diagnostic investigations. Also intraoperatively, it pointed; out high-risk nodules more accurately. Therefore, radio (Sestamibi) guided surgery could have an; interesting rule in the thyroid follicular lesion treatment.

๑ 2017 IJS Publishing Group Ltd. Published by Elsevier Ltd. All rights reserved.

\section{Introduction}

Nowadays, the characterization of the surgical pathology of the thyroid, both in benign (85-90\% of cases) and malignant (10-15\% of cases) diseases, employs traditional biochemical tests together with standardized ultrasound and scintigraphy examinations, which can give their assistance in obtaining a better cytology by guided needle aspiration, according to well established diagnostic and therapeutic criteria. On the other hand, the therapeutic setting of thyroid nodules defined by cytology as "follicular proliferation" or "follicular neoplasm", that represent between 10 and $20 \%$ of needle aspirations, still requires a diagnostic aimed surgery [1-3]. Even if liquid-based-cytology LBC-processed biopsies are a valid alternative to conventional cytology [4], the possibility of applying additional techniques to enhance the efficacy of the cytological diagnosis of thyroid nodules still cannot definitively solve the problem. In fact, in such situations, surgical strategy is influenced by the lack of suitable cytological criteria to distinguish follicular carcinomas from adenomas, requiring surgery to fully assess pathology. Here we report our experience in managing follicular neoplasms utilizing, in addition to the traditional surveys, both scintigraphy tracers and radio guided surgery.

\subsection{Objectives}

To evaluate the possible role of scintigraphy with positive indicators in thyroid nodular disease, both in preoperative diagnosis and in leading intraoperative surgical excision.

\section{Materials and methods}

From January 2006 to December 2013, we collected 4482 patients surgically treated for thyroid disease. Of these, 360 reported follicular neoplasms, to which we applied the following diagnostic flowchart [Table 1]:

In this flowchart we suggest using scintigraphy with sestamibi in the diagnostic pathway, with a procedural protocol described hereinafter.

\subsection{Scintigraphy procedure}

In patients previously undergone scintigraphy with pertechnetate, scintigraphy Whole-body scan images are acquired in supine position, both in front and at the back, and focused on the cervicothoracic region, at $10^{\prime}$ and $120^{\prime}$ (early and late acquisitions) after the intravenous administration of $555 \mathrm{MBq}$ of $99 \mathrm{~m}$ Tc-MIBI. A wide field gamma camera is used, provided with a low energy parallelhole collimator, interfaced by a dedicated workstation.

Sestamibi images are evaluated for each patient and directly compared to those with pertechnetate. In each patient, the number and the location of the nodules were also established on the basis of the clinical examination and of the ultrasound findings, besides Eco-Color-Doppler.

\subsection{Findings}

The $99 \mathrm{~m}$ Tc-MIBI scintigraphy shows a radiopharmaceutical uptake of both single nodules and in the context of multinodular goiters, colloid and neoplastic, benign or malignant; however, the latter acquisition (two hours after administrating the tracer) shows how the different tracer washout by both of the labeled drugs allows to better define the two morbid conditions. Also, over several experiments we noticed that colloid nodules had a late washout, whereas solid nodules, either benign (follicular adenoma) or malignant (carcinoma) [5-7], had an early washout.

According to our diagnostic algorithm, we enrolled our patients, $192 \mathrm{~F}$ and $168 \mathrm{M}$, mean age 44.5 years, suffering from thyroid nodular disease, and "follicular neoplasm" carriers at the FNAB. Of these patients, 156 patients had a single nodule, "cold" on scintigraphy with $99 \mathrm{mTc}$-pertechnetate and Eco-Color-Doppler intranodular vascularization (Pattern III sec. Lagalla), while the remaining 204 patients had a multinodular disease, although FNAB was carried out on the most suspicious nodules according to the findings on ultrasonography and scintigraphy. All patients underwent a preoperative $99 \mathrm{~m}$ Tc-MIBI scintigraphy (iv administration of 555MBq $99 \mathrm{~m} \mathrm{Tc}-\mathrm{MIBI})$, and early (10 min) and late ( $2 \mathrm{~h}$ ) images were acquired; sestaMIBI images were obtained for each patient and directly compared to those obtained by $99 \mathrm{mTc}$-pertechnetate. Then, immediately before surgery, following the iv administration of a tracer dose $(37 \mathrm{mBq})$ of sestamibi, we used a probe for radio guided surgery (Neoprobe) in order to perform a more rigorous selection of the most uptaking nodules. This probe is composed of a stylus-like detector and of a digital console showing the tracer uptake as percentage [Fig. 1].

The intraoperative pathological examination was not performed, because of the limitations above mentioned. Once removed, and always using the same radio guided probe, the excised sample was immediately examined by a pathologist, who accurately examined it by practicing serial slices of the most uptaking lesions [Fig. 2].

We undertook 324 total thyroidectomies and 36 hemi thyroidectomies, in patients with one single node and a macroscopically intact contralateral lobe [Table 2].

\section{Results}

By means of preoperative scintigraphy, we distinguished:

- 120 low up-take nodules (of which only 6 as single nodes) with an uptake until $12 \mathrm{~Gy}$;

- 144 medium up-take nodules with an uptake until 24 Gy;

- 96 high up-take nodules (including 72as single nodes) with an uptake over $24 \mathrm{~Gy}$.

Intraoperative survey confirmed, as percentage, the preoperative findings, and the high uptake in the reported 96 nodules; particularly, in one case, the high concentration of the tracer was related to the presence of a small nodule ( $<1 \mathrm{~cm}$ diameter), not revealed at preoperative examinations, and placed behind a bigger 
Table 1

Diagnostic flowchart of thyroid nodules.

\section{Thyroid Nodule Treatment}

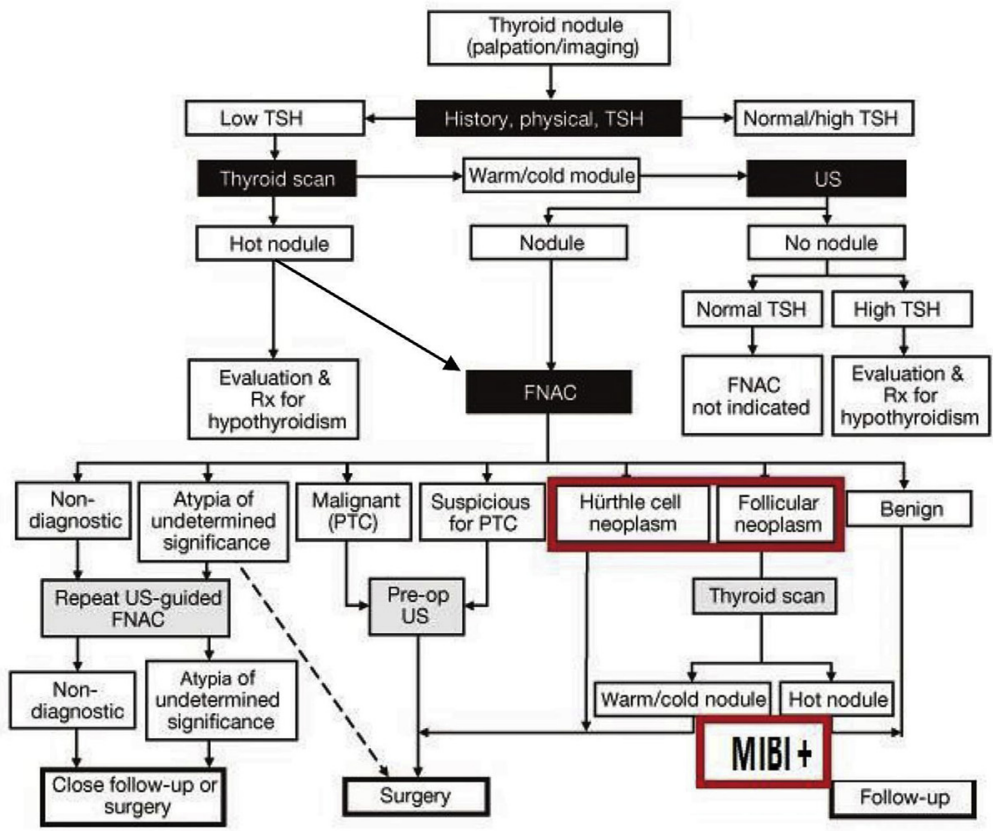

FNAC Classification

\begin{tabular}{|c|l|c|l|l|}
\hline \multicolumn{2}{|l|}{ Bethesda diagnostic category } & \multicolumn{2}{l|}{ British Thyroid Association } & American Thyroid Association \\
\hline \hline I & Non-diagnostic or unsatisfactory & Thy1 & Non-diagnostic & Non-diagnostic/unsatisfactory \\
\hline II & Benign & Thy2 & Non-neoplastic & Benign \\
\hline III & $\begin{array}{l}\text { Atypia of undetermined } \\
\text { significance or follicular lesion of } \\
\text { undetermined significance }\end{array}$ & Thy3a & Atypical features present & Indeterminate or suspicious for \\
IV & $\begin{array}{l}\text { Follicular neoplasm or suspicious } \\
\text { for a follicular neoplasm }\end{array}$ & Thy3f & Follicular neoplasm & Indeterminate or suspicious for \\
malignancy
\end{tabular}

Table 1 Diagnostic flowchart of Thyroid Nodules

In the flowchart it's red stressed the points where our methodology is suggested

nodule with a mean uptake. Pathology examination reported the following conditions (Graphic no 1):

- 60 cases of benign nodules in colloid goiter;

- 96 cases of micro-follicular adenoma in one nodule;

- 120 cases of micro-follicular adenoma in a multinodular goiter;

- 60 cases of follicular carcinoma (6 of which T1);

- 24 cases of papillary carcinoma (3 of which as "microcarcinoma").
From a comparative analysis, between preoperative and intraoperative findings, together with histological reports, we concluded that (Graphic no 2):

- Scintigraphy findings and washout ratios were significantly different in patients with thyroid neoplasms (follicular adenoma or follicular carcinoma) than in those with colloid goiter, which confirms a low intraoperative uptake in all of the 60 cases clinically defined as lumps in a colloid goiter; this reveals and 


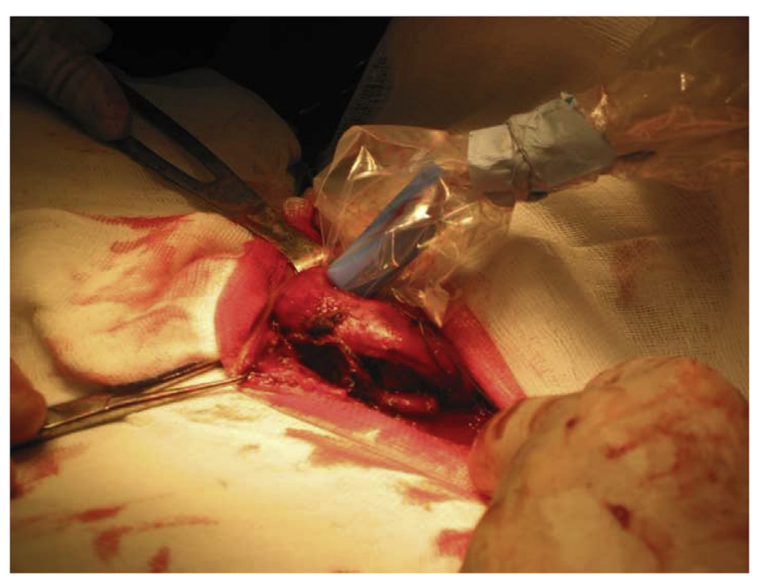

Fig. 1. Intraoperative findings by Neoprobe.

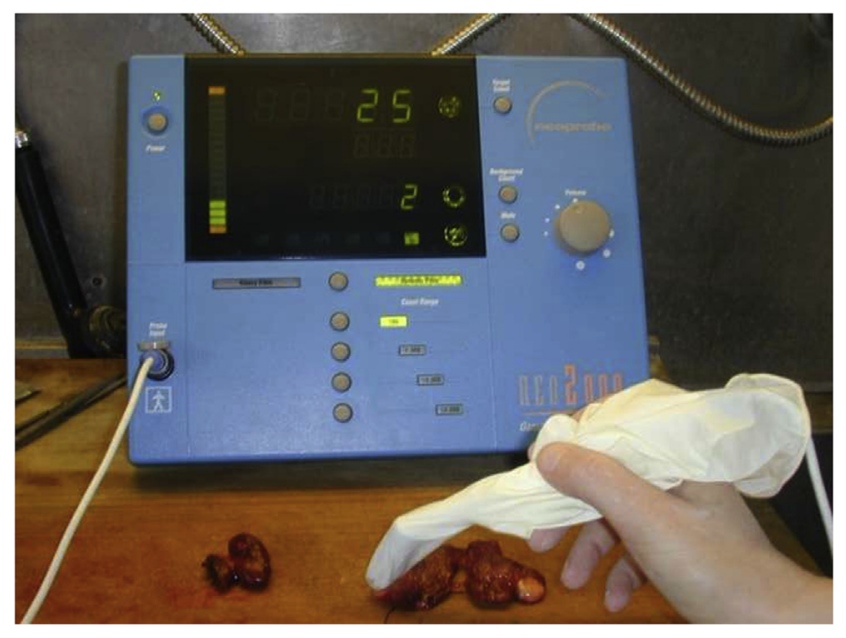

Fig. 2. Uptake determination on a surgical sample.

confirms also the higher specificity of sestamibi in relation to Ecocolordoppler in the differential diagnosis between follicular neoplasms and goiter;

- The 60 follicular carcinomas, appearing as nodules, had a high preoperative uptake and the highest values, as percentage of intraoperative uptake, in 48 cases, with a mean value (37.2 Gy) about 4 times greater than colloid parenchyma ( $9,7 \mathrm{~Gy}) \mathrm{p}<0.01$; this suggested a possible and effective role as "positive indicator" of $99 \mathrm{~m}$ Tc-MIBI for malignant follicular neoplasms;

- The nodules reported as "micro-follicular adenomas" had intermediate values due to the mean up-take both in preoperative and intraoperative phases (however, in 36 cases, an high uptake was present) with mean values of uptake (24.8 Gy) at least double compared to colloid parenchyma (9.7 Gy) p $<0.01$;

Certainly, we cannot draw proper conclusions about the present role of sestamibi for this lumps category, therefore, the value so unique and essential of microscopic examination for differential

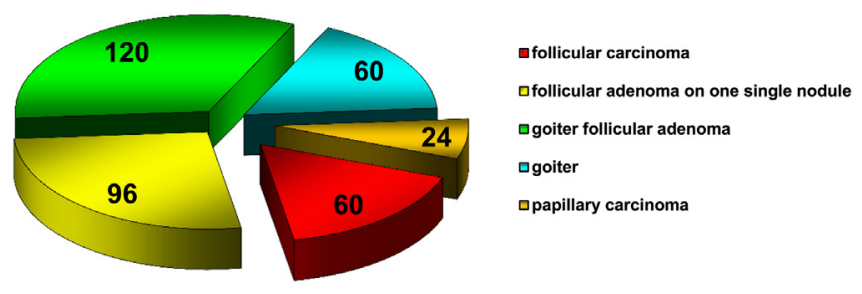

Graph no 1. Pathologic subsets of the examined cases.

diagnosis still remains unchanged.

However, it is useful to remark some highlighted evidences:

- In 3 of the above mentioned cases, the intraoperative radio guided search revealed a highly up-taking lump (uptake index of 42), missed at the preoperative diagnosis, as it was partly hided by an adjacent larger nodule, with a mean uptake instead; in this patient, who also presented a normal contralateral lobe, we chose, on the basis of these considerations, to undertake a total thyroidectomy; eventually, the definitive pathological examination showed an "atypical follicular adenoma" $(>1 \mathrm{~cm})$, inside a micro/macro-follicular goiter (classified as a true follicular carcinoma $\mathrm{T} 1$ by various Authors). This suggests and confirms the usefulness of our technique also as a guide to the most appropriate surgical strategy.

- The proved 24 cases of "papillary carcinoma" had only a mild uptake (13.3 mean value) in the preoperative survey, and the following intraoperative investigation revealed no higher rates of uptake as well; this suggests and confirms the role of sestamibi as a cellular tracer, with follicular structures electively accumulating the tracer.

All the 144 patients having only one node and choosing hemithyroidectomies surgical option reported a low uptake in 24 cases (16 average), and a high uptake in 12 cases (22); all 36 suffered from micro-follicular adenoma, that is why it was not necessary to further extend the surgical removal.

\section{Discussion}

Even if all authors agree on the necessity of the surgical removal of suspicious lumps, however not everyone agree that total thyroidectomy is required. Moreover, according to most studies, a definitive diagnosis cannot be established by an intraoperative pathologic examination, since it does not seem to offer greater diagnostic possibilities than cytology (lacking samples on all the sections, lacking a proper study of capsule and of vascular invasion) $[8,9]$. Therefore, this creates the conditions for a difficult choice, suggested by several evaluations, which lead some surgeons to prefer total thyroidectomy for all histological-types of follicular proliferation, regardless of the contralateral lobe conditions, while others restrict the intervention to the removal of lumps affectedlobe, supported by recent experiences with minimally invasive surgical approaches [10], obviously given that contralateral lobe exploration and intraoperative ultrasound are normal, and leaving the radicalization to a second surgical intervention in case of malignancy from definitive pathological examination. Both of the

Table 2

Demographic and clinical characteristics of cohort. Single cold nodule (SCN), Multinodular disease (MD), Total thyroidectomy (TT), Hemi-Thyroidectomy (HT).

\begin{tabular}{|c|c|c|c|c|c|c|}
\hline M & $\mathrm{F}$ & Mean age & $\mathrm{SCN}$ & MD & TT & HT \\
\hline 168 & 192 & 44.5 years & 156 & 204 & $324(144 \mathrm{SCN}+180 \mathrm{MD})$ & $36(12 \mathrm{SCN}+24 \mathrm{MD})$ \\
\hline
\end{tabular}




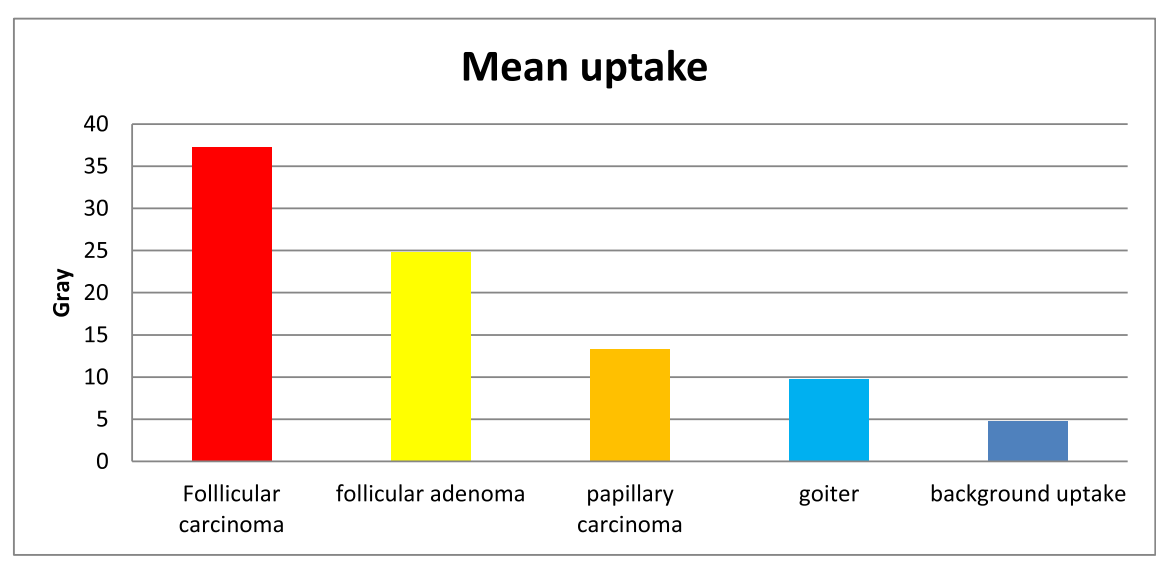

Graph no 2. Mean uptake subsets.

choices are obviously questionable. Given the low probability that "follicular proliferation" is the term for a pathologically documented cancer (less than 15-20\%), it appears excessive, according to some authors, to prefer total thyroidectomy in case of a single nodule $[11,12]$. On the other hand, even if in many studies it does not demonstrate morbidity increase, the two-stage operation, desirable in the short time, is experienced by the patient as a stressful condition, and it increases the costs for the hospital permanence. Obviously, in case of "follicular neoplasm", found in a multinodular goiter, the question is less important, since the current surgical option for the multinodular pathology is still the total thyroidectomy, considered as the "gold standard" for the treatment of benign multinodular disease, euthyroid or thyrotoxic, as well as for tumors [13-15]. Supporters of the "two times" strategy include, among the reasons for their choice, the increased risk of complications (laryngeal injuries, permanent hypoparathyroidism), higher than in case of unilateral intervention. Nonetheless, there are a lot of contributions in the surgical literature about the incidence of complications after total thyroidectomy, in practice similar, in experienced hands, to unilateral surgery. Moreover, about cancers, there is no doubt about the therapeutic superiority of total thyroidectomy, especially due to some types of cancer features (multifocality and bilaterality, especially for papillary carcinoma), to the possibility of a proper follow-up in absence of uptaking thyroid tissue (possibility of performing total-body scintigraphy, role of thyroglobulin as a tissue marker), to the therapeutic chances offered by ablative radioiodotherapy with lower doses of 131-I and to adequate treatment in case of metastases, and finally because to nonnegligible risks, in many law suits, related to reoperation [16,17]. All of these reasons would result in a better long-term prognosis. Therefore, total thyroidectomy should be considered as the eligible surgical treatment and, moreover, it should be desirable to "centralize" the treatment of these neoplasms in surgical centers, which can hold permanent complication rates under the percentage of 1-2\%. Actually, particularly in AngloSaxon countries there is a discussion on the possibility of less extensive excisions (hemi thyroidectomy), less burdened by postoperative consequences for what concerns T1 tumors in follicular phase (well differentiated tumors of size equal to or smaller than 1 centimeter without capsular invasion). This option should be weighted for those patients previously surgically treated for benign disease, and for the ones whose definitive pathological examination reported a follicular thyroid cancer, following an appropriate assessment of the prognostic criteria: smaller than one centimeter, female gender, age $<45$ years, absence of capsular invasion [18].

The main limitation of this study were the retrospective method of analysis and the absence of the control group.

\section{Conclusions}

Actually, the large amount of patients with thyroid nodular disease requires an accurate diagnostic definition for a successful therapeutic strategy. After clinical diagnosis and medical history, we agree on the undisputed role and utility of both ultrasound and FNAB in selecting, among the patients with nodular disease, those to be addressed to surgery, in order to reduce the number of unnecessary interventions $[19,20]$. On the other hand, the planned therapy for nodules recognized as "follicular neoplasm" after FNAB is doubtful. In particular, about the lesions, the criteria for a differential diagnosis between follicular adenoma and well differentiated carcinoma are not cytological but histological, and consequently the surgical excision for a definitive diagnosis is absolutely necessary. Given the diagnosis of follicular neoplasms by cytology, the main problem is the thyroidectomy extent, because the information achieved by intraoperative examination is poor, which is not useful to solve all doubts. The present study evaluated the role of scintigraphy with positive indicators (99 m Tc-MIBI) during both preoperative differential diagnosis and intraoperatively as a guide to surgical strategy. The preoperative phase has confirmed the usefulness of such a qualitative and semiquantitative assessment technique in differentiating colloid goiters from thyroid neoplasms, thereby improving the information already obtained by ultrasound techniques, and allowing a more conservative surgical approach, where possible (single nodule with not nodular contralateral lobe), while, in both benign and obviously malignant multinodular diseases, total thyroidectomy is the "gold standard" technique. In perspective, also preoperatively, this method can be proposed in multinodular diseases and in other selected cases, in order to allow a more accurate differentiation of clinical and sonographic suspected nodularity requiring a cytological examination, and to improve the FNAB specificity and accuracy. In our experience, the use of a tracer dose of preoperative sestamibi, and of a probe for intraoperative radio guided surgery, together with the values provided by uptake rates, emphasized the level of suspected carcinomas in nodules that turned out to be malignant after histology, thus contributing to a better definition of the surgical strategy. In the context of benign neoplastic disease (micro-follicular adenomas), instead, the method confirmed its acknowledged limits, and the consequent necessity of a definitive diagnosis given by pathology. In such cases, the likely surgical overtreatment will be surely limited to one neoplastic lump, since the use of total thyroidectomy is widely accepted in multinodular 
forms [18]. Therefore, we hope to achieve, through other studies, especially through molecular biology and genetics [21], the possibility of identifying new biochemical and genetic instrumental parameters (our described method seems to offer interesting perspectives) able to preoperatively identify those patients at high risk of cancer who can be effectively treated through surgery, thus avoiding overtreatments, and the law suits which these are related to, as well. In our experience, we are developing the same methodology to identify lymph nodal follicular metastases by assessing the intraoperative uptake of MIBI tracer [22,23]. In the meanwhile, the most reliable diagnosis for these patients is achieved through the surgical pathology, especially when the diagnosis of microcarcinoma seems a more reliable objective [24].

\section{Ethical approval}

Not needed.

\section{Funding}

This research did not receive any specific grant from funding agencies in the public, commercial, or not-for-profit sectors.

\section{Author contribution}

Please specify the contribution of each author to the paper, e.g. study design, data collections, data analysis, writing. Others, who have contributed in other ways should be listed as contributors.

PD, RR, DG are the main surgeon of the Division: they performed 95 of the 360 Thyroidectomies procedures for Thyroid Follicular Lesions.

PD: main author, agree to be accountable for all aspects of the work in ensuring that questions related to the accuracy or integrity of any part of the work are appropriately investigated and resolved. He has designed the original study, the diagnostic and therapeutic flowchart and finally he has ideated the technical innovation.

CG: has made substantial contributions to conception and design, acquisition of data, analysis and interpretation of data. He has controlled that data from different Surgical Units were over imposable.

RP: has made substantial contributions to conception and design, acquisition of data, analysis and interpretation of data. He has controlled that data from different Surgical Units were over imposable.

AP: has made substantial contributions to conception and design, acquisition of data, analysis and interpretation of data. He has controlled that data from different Surgical Units were over imposable.

TS: has made substantial contributions to conception and design, acquisition of data, analysis and interpretation of data. He has controlled that data from different Surgical Units were over imposable.

DL: Director of the Division and of the Department, he has been involved in drafting the manuscript, revising it critically for important intellectual content and specially he has given final approval of the version to be published.

DFM: he is one of the main surgeon of the Unit, he has performed 85 of the 360 thyroidectomies procedures for Thyroid Follicular Lesions.

CG: he is one of the main surgeon of the Unit, he has performed 73 of the 360 thyroidectomies procedures for Thyroid Follicular Lesions.

CR, AN: They are the main surgeon of the Division: they performed 77 of the 360 Thyroidectomies procedures for Thyroid Follicular Lesions.
SA: he is one of the main surgeon of the Unit, he has performed 30 of the 360 thyroidectomies procedures for Thyroid Follicular Lesions.

$\mathrm{CV}$ : he is the main researcher in Nuclear Medicine, he has coordinated the radiological image acquisition database and he has controlled any aspects of the radio guided procedures, training all the nuclear medicine techniques and checking uptake values and their classification.

AM: she is the main researcher in Pathology, she has coordinated all the cytological and histological exams, controlling the right acquisition, methodology and classification.

$\mathrm{BV}$ : he is the main researcher in Endocrinology, he has coordinated all endocrine aspects from patients recruitment to their postoperative follow up, giving an important contribute to the flowchart respect.

\section{Conflicts of interest}

None.

\section{Guarantor}

Giovanni Conzo.

Domenico Parmeggiani.

\section{Research registration unique identifying number (UIN)}

researchregistry2007.

\section{References}

[1] K. Kakudo, Y. Bai, Z. Liu, Y. Li, Y. Ito, T. Ozaki, Classification of thyroid follicular cell tumors: with special reference to borderline lesions, Endocr. J. 59 (2012 $1-12$.

[2] C.R. McHenry, R. Phitayakorn, Follicular adenoma and carcinoma of the thyroid gland, Oncologist 16 (2011) 585-593.

[3] A. Bastagli, L. De Pasquale, L. Shubert, Approccio chirurgico alla proliferazione della tiroide, Atti Soc. It. Chir. III (2000) 13-16.

[4] G. Fadda, E.D. Rossi, Liquid-based cytology in fine-needle aspiration biopsies of the thyroid Gland, Acta. Cytol. 55 (2011) 389-400.

[5] S. Muller, B. Guth-Tougelides, H. Creutzig, Imaging of malignant tumors with Tc-99m MIBI SPECT, J. Nucl. Med. 28 (1987) 562.

[6] E. Kresnik, H. Galoowitsch, P. Mikosch, M. Molnar, W. Pipam, I. Gomez, P. Lind Evaluation of thyroid nodules with technetium 99m tetrofosmin dual phase scintigraphy, Eur. J. Nucl. Med. 24 (1997) 716-772.

[7] C. Aktolun, H. Baylan, M. Kir, Clinical experience with Tc99m-MIBI imaging in patients with malignant tumors: preliminary results and comparison with $\mathrm{Tl}-$ 201. Clin. Nucl. Med, 17 (1992) 171-176.

[8] F. Monzani, M. Caraccio, P. Iacconi, P. Faviana, P. Miccoli, Prevalence of cance in follicular thyroid nodules: is there still a role for intraoperative frozen section analysis? Thyroid 13 (2003) 389-484.

[9] P. Miccoli, R. Elisei, G. Materazzi, M. Capezzone, P. Berti, A. Pinchera, Minimally invasive videoassisted thyroidectomy for papillary carcinoma: a prospective study of its completeness, Surgery 132 (2002) 1070-1074.

[10] R. Cirocchi, C. Boselli, S. Guarino, A. Sanguinetti, S. Trastulli, J. Desiderio, A. Santoro, F. Rondelli, G. Conzo, D. Parmeggiani, G. Noya, G. De Toma N. Avenia, Total thyroidectomy with ultrasonic dissector for cancer: multicentric experience, World J. Surg. Oncol. 27 (2012) 10, 70.

[11] K. Van Nguyen, R.A. Dilawari, Predective of values AMES scoring system in selection of extent of surgery in well differentiated carcinoma of thyroid, Am. Surg. 61 (1995) 151-155.

[12] A. Pezzolla, G. Docimo, R. Ruggiero, M. Monacelli, R. Cirocchi, D. Parmeggiani, G. Conzo, A. Gubitosi, S. Lattarulo, A. Ciampolillo, N. Avenia, L. Docimo N. Palasciano, Incidental thyroid carcinoma: a multicentric experience Recenti Prog. Med. 101 (2010) 194-198.

[13] Q. Liu, G. Djuricin, R.A. Prinz, Total thyrodectomy for benign thyroid disease Surgery 123 (1997) 2-7.

[14] T.S. Reeve, Total thyroidectomy :the preferred option for the multinodular goiter, Ann. Surg. 206 (1987) 782-786.

[15] J.R. Brooks, H.F. Starnes, D.C. Brooks, Surgical therapy of thyroid carcinoma. A review of 1249 solitary thyroid nodules, Surgery 104 (1988) 904-906.

[16] M. De Falco, G. Oliva, M. Ragusa, C. Misso jr, D. Parmeggiani, P. Sperlongano, F. Calzolari, E. Puxeddu, C. Misso, L.A. Marzano, A. Barbarisi, U. Parmeggiani, N. Avenia, Surgical treatment of differentiated thyroid carcinoma: a retrospective study, G. Chir. 29 (2008) 152-158. 
[17] U. Parmeggiani, D. Parmeggiani, C. Giudicianni, M. De Falco, Carcinoma differenziato della tiroide. Prognosi e terapia, Minerva Chir. 56 (2001) 583-591.

[18] S.E. Kountakis, I.G. Skoulas, A.A. Maillard, The radiologic work-up in thyroid surgery: fine needle biopsy versus scintigrafy and ultrasound, Ear Nose Throat. J. 81 (2002) 151-154.

[19] K. Demirel, O. Kapucu, C. Yucel, H. Ozdemir, G. Ayvaz, F. Taneri, A Comparison of Radionuclide Thyroid Angiography, Tc-99m MIBI Scintigraphy and Power Doppler Ultrasonography in the Differential Diagnosis of Solitary Cold Thyroid Nodules, Verlag, 2003.

[20] A. Bartolazzi, A. Gasbarri, M. Papotti, G. Bussolati, T. Lucante, A. Khan, H. Inohara, R. Tecce, O. Larsson, et al., Application of an immunodiagnostic method for improving preoperative diagnosis of nodular thyroid lesions, Lancet 357 (2001) 1644-1650.

[21] E.L. Chua, L. Young, W.M. Wu, J.R. Turtle, Q. Dong, Cloning of TC-1, a nove gene found to be overexpressed in thyroid cancer, Genomics 69 (2000) $342-347$
[22] G. Conzo, P.G. Calò, A.A. Sinisi, A. De Bellis, D. Pasquali, S. Iorio, E. Tartaglia, C. Mauriello, C. Gambardella, F. Cavallo, F. Medas, A. Polistena, L. Santini, N. Avenia, Impact of prophylactic central compartment neck dissection on locoregional recurrence of differentiated thyroid cancer in clinically nodenegative patients: a retrospective study of a large clinical series, Surgery 155 (6) (2014) 998-1005.

[23] G. Conzo, N. Avenia, G. Bellastella, G. Candela, A. de Bellis, K. Esposito, D. Pasquali, A. Polistena, L. Santini, A.A. Sinisi, The role of surgery in the current management of differentiated thyroid cancer, Endocrine 47 (2) (2014) $380-388$.

[24] P.G. Calò, G. Conzo, M. Raffaelli, F. Medas, C. Gambardella, C. De Crea, L. Gordini, R. Patrone, L. Sessa, E. Erdas, E. Tartaglia, C.P. Lombardi, Total thyroidectomy alone versus ipsilateral versus bilateral prophylactic central neck dissection in clinically node-negative differentiated thyroid carcinoma. A retrospective multicenter study, Eur. J. Surg. Oncol. 43 (2017) 126-132. 